\title{
Analysis of the external factors' effect on optimized profile of a transporter's oblique end
}

\author{
Alexey Dyachenko, ${ }^{1, *}$ and Tatiana Savostina ${ }^{1}$ \\ ${ }^{1}$ Don State Technical University, 344003, Gagarin sq., 1, Rostov on Don, Russia
}

\begin{abstract}
The present article is oriented on development of engineering and design parameters of a threshing separator designed as hyperboloid of revolution of one nappe for grain crop harvesting. Dependence of optimized cutting profiles on physical and mechanical properties of grain mass and parameters of a threshing separator is defined to ensure uniform supply of grain mass to threshing. The mathematical model of the profile defining process is developed to ensure uniform supply of grain mass to threshing.
\end{abstract}

\section{Introduction}

A large proportion of losses in the process of grain-harvesting is a grain loss in a threshing separator as this unit is the main threshing and separating part of a combine harvester.

Research hypothesis. Efficiency of the threshing and separating process in a threshing separator may be achieved by means of re-engineering of a feed unit designed to ensure a uniform layer of grain mass in a concave that makes it possible to mitigate the losses in the form of returns and seeds damaged during threshing.

Goal of the research - improvement of effectiveness of grain mass threshing and separating in a threshing separator designed as hyperboloid of revolution of one nappe for grain crop harvesting together with the rationale for effect of external factors on grain mass supply uniformity.

Tasks of the research - on the basis of experimental studies, to find main directions for design development of a threshing separator, to evaluate the effect of external factors on threshing and separating performance quality.

The research is concerned with a threshing separator designed as hyperboloid of revolution of one nappe.

Methodology and research methods. In the course of experimental and theoretical studies conducted by applying the universal laws of mathematics and physics, experimental data were obtained in a laboratory environment. When processing the received data, common methods and approaches to multifactorial experiment design were used in accordance with current GOSTs for data processing and methods of mathematical statistics.

Theoretical part. The main function of threshing is disruption of the link between a grain and an ear, but this link depends on various factors such as grain mass humidity at the

*Corresponding author: Alexey-a2@mail.ru 
moment of harvesting, ear texture, maturity, and dimensions. This research was conducted in a lab environment using specially designed laboratory machines and on-farm using experimental units [1].In the context of disruption of the link between a grain and an ear, it was found that multiple stress on grain mass disrupts the link between a grain and an ear. Separation of a grain and an ear rises with an increase in impact frequency, but at high speeds [3] there is a reduction [4] in the quality of a grain and excessive grain shattering [5].

A crucial aspect is that every variety of wheat has its own characteristics related to impact speed; it results in disruption of the link between a grain and an ear [6,7].The humidity value at the moment of harvesting is volatile between $4 \%$ and $24 \%[8,9]$, and it also affects separation of a grain from an ear [10].

Microdamages change physical and mechanical properties of grain little, if at all, but they degrade the quality of seed material [11]. Wheat grains having microdamages cannot be separated from that not damaged even using state-of-the-art equipment. That is why the challenge for modern equipment is to reduce damages of grains at the moment of harvesting [12].

The ideal condition for threshing is a process, which eliminates losses related to returns, straw chopping and grain shattering [13]. To achieve it, it is necessary to arrange a uniform grain supply evenly distributed along the width of a threshing separator [14]. A laboratory experimental machine (fig. 1) is designed for late separation of grain mass flow and provides for optimal uniformity of grain mass flow when it is supplied to threshing [15].

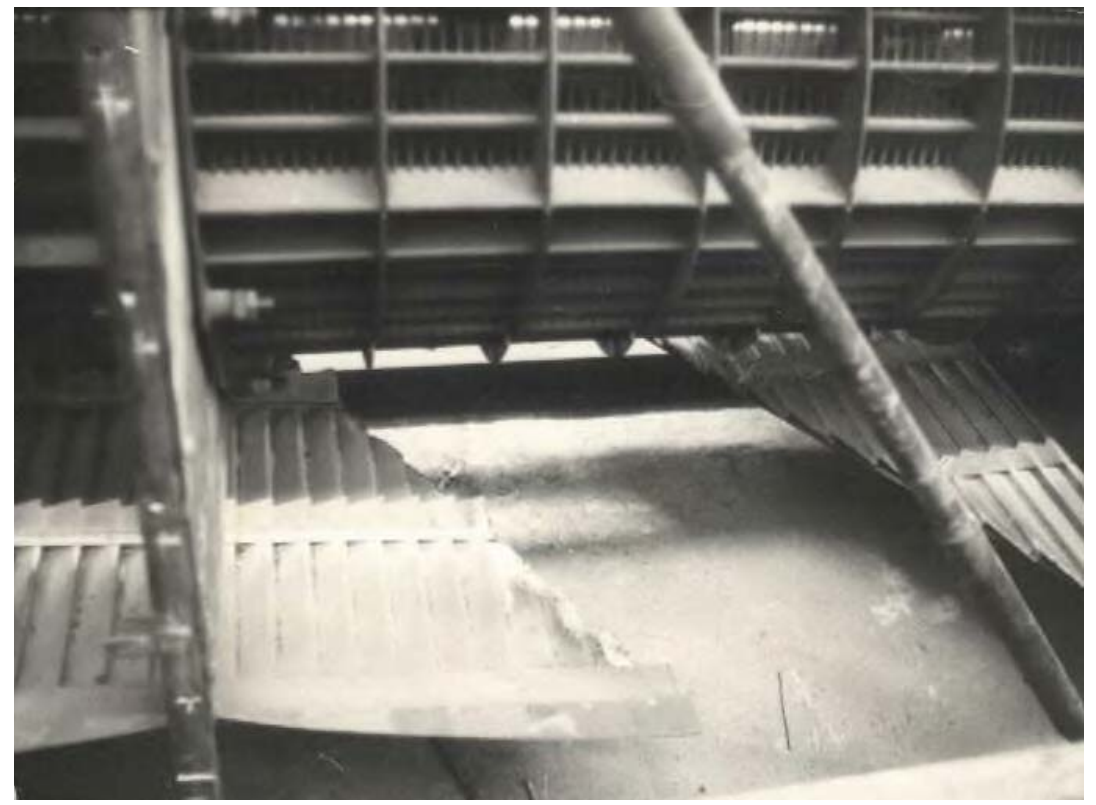

Fig. 1. A laboratory machine equipped with a transporter with oblique ends

The laboratory machine represented on fig. 1 consists of a frame, an electric motor, a rotor designed as hyperboloid of revolution of one nappe with a bar, an oblique-ended transporting board for grain supply, and hinged zoned cells for grain collection.

Separation of macrodamaged grains from whole grains occurs in each hinged zoned cell. Separated macrodamaged grains are weighted. Average across two experiments is reported.

Steps of the experiment. 
1. To prepare a laboratory machine for the experiment, to test it in various modes. To charge grain mass into a feeding device.

2.To conduct tests at different humidity rate of grains: $8 \%, 12 \%, 16 \%, 20 \%$ and $24 \%$.

3.To calculate the quantity of threshed and microdamaged grains after every test.

The laboratory experimental tests are conducted three times for every humidity rate ranging from $8 \%$ to $24 \%$.

The analysis of the found patterns of threshing quality indicators shows that with an increase in grain mass humidity aggregate separation of grains decreases to a small extent (fig. 2).

The diagram shows that with an increase in grain mass humidity from $8 \%$ to $24 \%$ aggregate separation of grains by a thresher decreases to a small extent. This is explained by the fact that in a tangentially axial threshing separator designed as hyperboloid of revolution of one nappe, grain mass runs a longer way in a threshing channel and stays under pressure of threshing units for a longer time.

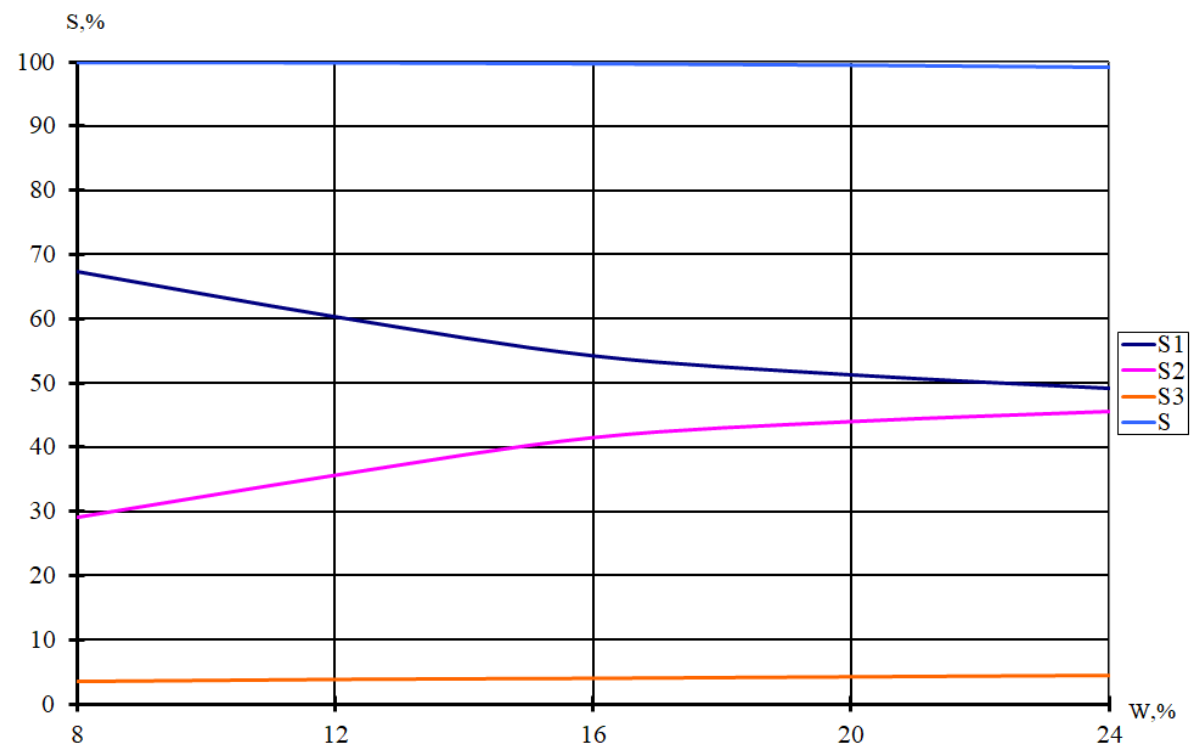

Fig. 2.Grain separation vs humidity in various zones

There is a decrease in grain separation in the first zone, but this decrease is compensated by an increase in grain separation in the second and third zones (fig. 2).The diagram also shows that an increase in humidity results in threshing ability degradation and in an increase in returns.

Dockage of the grain separated by a thresher is decreased when humidity rises (fig. 3), as straw is less chopped and there is less small tailings. 


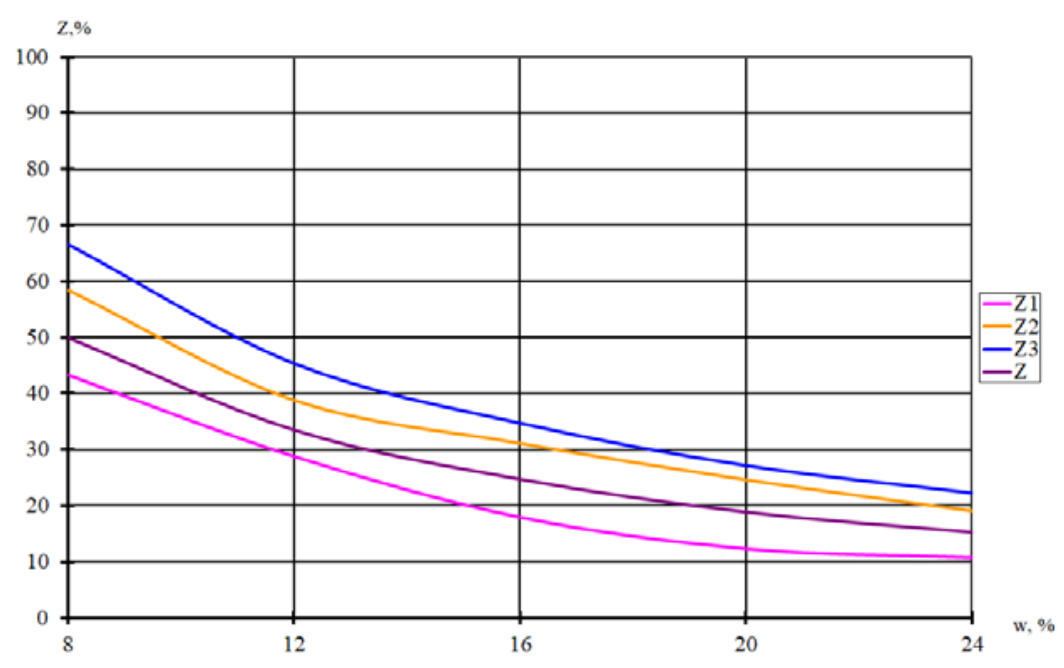

Fig. 3. Grain dockage vs humidity in various zones

With an increase in humidity of the threshed mass, grain dockage separated by a thresher is decreasing both in total and over the length of a roller (zonally) (fig.3).This is explained by the fact that an increase in humidity of the threshed mass results in degradation of chop ability of culms.

When humidity rises from 8 to $20 \%$, returns rise from $0.1 \%$ to $0.37 \%$.It should be noted that an increase in humidity of the threshed mass results in a rise of loss rate related to returns. For example, returns rise just by $0.02 \%$ with the increase in humidity from 8 to $12 \%$, however returns rise by $0.18 \%$ with the increase in humidity from 20 to $24 \%$.

\section{Materials and methods}

Development of a mathematical and numerical model is a process oriented on search of the independent variable. The numerical method is to approximate the function of $f(x)$ by the approximate function of $\varphi(x)$, values of which differ from the experimental data in the range under investigation to a small extent $-f(x) \approx \varphi(x)$. The received approximate functional dependency based on processing of the experimental data is known as an approximative function.

To write an equation of regression is to define its parameters. To define regression parameters let's use the least-squares method. This method enables to define parameters, at which sum of squared errors is minimum with regard to assumed findings. The principle of the numerical method is to find the key factors affecting a variable. We take a linear approximation as an approximate function.

The empirical formula of a linear function is as follows:

$$
\varphi(x, a, b)=a x+b
$$

where $\varphi-$ a known mathematical linear function,

$a$ and $b$ - unknown parameters.

The task is to determine parameters of $a$ u $b$, so that their values of approximate function match as closely as possible against values of the function under study in points of $x_{i}$. The difference between these errors is represented by means of the parameter of $\varepsilon_{i}$. 
The sum of squared errors is written down as follows:

$$
S=S(a, b)=\sum_{i=1}^{n} \varepsilon_{i}^{2}=\sum_{i=1}^{n}\left[\varphi\left(x_{i}\right)-y_{i}\right]^{2}=\sum_{i=1}^{n}\left(a x_{i}+b-y_{i}\right)^{2} \rightarrow \min
$$

To define values of $a$ and $b$, it is necessary to define a minimum of the function of $S(a, b)$.To meet the required condition for this minimum for the function of $S$ :

$$
\left\{\begin{array}{l}
\frac{\partial S}{\partial a}=0 \\
\frac{\partial S}{\partial b}=0
\end{array}\right.
$$

or

$$
\left\{\begin{array}{c}
2 \sum_{i=1}^{n}\left(a x_{i}+b-y_{i}\right) x_{i}=0 \\
2 \sum_{i=1}^{n}\left(a x_{i}+b-y_{i}\right)=0
\end{array}\right.
$$

Let us simplify the received system:

$$
\left\{\begin{array}{c}
a \sum_{i=1}^{n} x_{i}^{2}+b \sum_{i=1}^{n} x_{i}=\sum_{i=1}^{n} x_{i} y_{i} \\
a \sum_{i=1}^{n} x_{i}+b n=\sum_{i=1}^{n} y_{i}
\end{array}\right.
$$

Let us introduce additional notations:

$$
\begin{aligned}
\mathrm{SX} & =\sum_{i=1}^{n} x_{i} \\
S X X & =\sum_{i=1}^{n} x_{i}{ }^{2} ; \\
\mathrm{SY} & =\sum_{i=1}^{n} y_{i} ; \\
S X Y & =\sum_{i=1}^{n} x_{i} y_{i} .
\end{aligned}
$$

We acquire a system of equations to find parameters of $a$ and $b$ :

$$
\left\{\begin{array}{c}
a \cdot S X X+b \cdot S X=S X Y \\
a \cdot S X+b \cdot n=S Y
\end{array}\right.
$$

Using (6) we find the parameters of $a$ и $b$ : 


$$
\begin{array}{r}
a=\frac{S X Y \cdot n-S X \cdot S Y}{S X X \cdot n-S X \cdot S X} ; \\
b=\frac{S X X \cdot S Y-S X \cdot S X Y}{S X X \cdot n-S X \cdot S X} .
\end{array}
$$

\section{Results and discussion}

It is necessary to approximate an unknown functional dependency between $X$ and $Y$, received as a result of the experiments, on the second feed rate value. Experimental data were tabulated (table 1).

Table 1. Experimental data

\begin{tabular}{|c|c|c|c|c|c|c|}
\hline $\boldsymbol{n}$ & $\mathbf{1}$ & $\mathbf{2}$ & $\mathbf{3}$ & $\mathbf{4}$ & $\mathbf{5}$ & $\mathbf{6}$ \\
\hline$X$ & 0 & 240 & 480 & 720 & 960 & 1200 \\
\hline$Y$ & 0 & 292.22 & 455.82 & 598.09 & 747.42 & 1000 \\
\hline
\end{tabular}

Let us draw points specified in the table 1 on the diagram to obtain a graphical format of the received dependence (fig. 4).

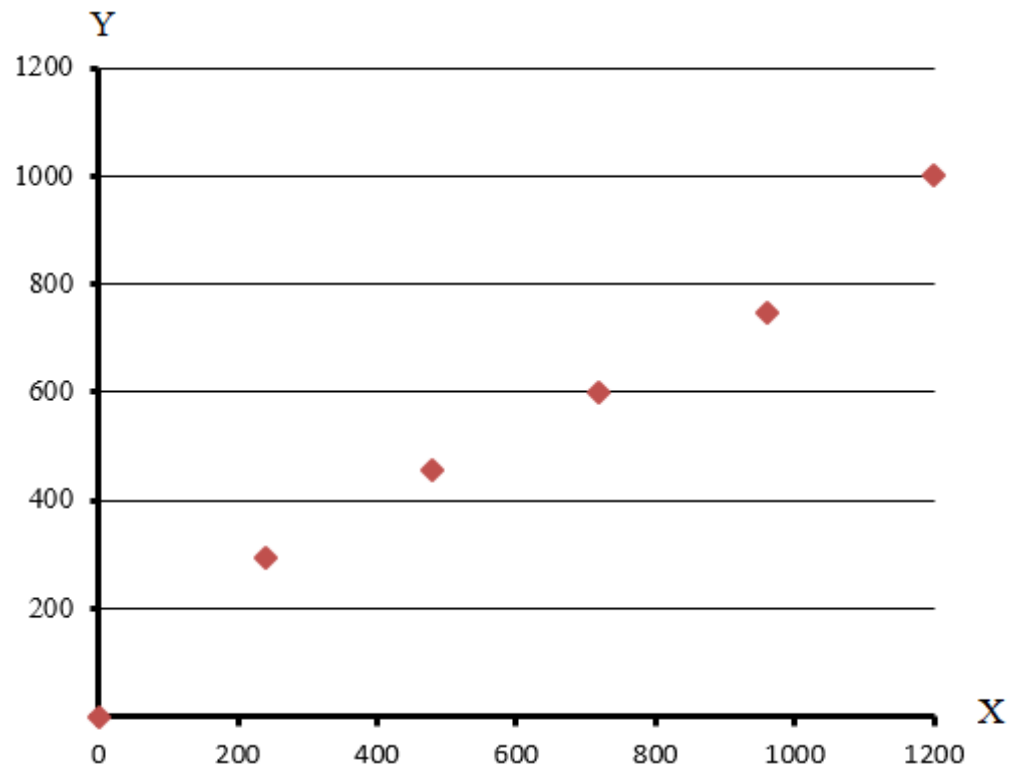

Fig. 4. Values of the experimental data

The diagram represented on the figure 4 shows that a linear equation of the form $f=a x$ $+b$, which governs experimental values as accurately as possible, may be applied as an approximative function.

Let us find the function's parameter values: $a$ and $b$ :

$$
\mathrm{SX}=\sum_{i=1}^{n} x_{i}=0+240+480+720+960+1200=3600
$$




$$
\begin{gathered}
S X X=\sum_{i=1}^{n} x_{i}{ }^{2}=0+240^{3}+480^{3}+720^{3}+960^{3}+1200^{2}=3168000 \\
S Y=\sum_{i=1}^{n} y_{i}=0+292,22+455,82+598,09+747,42+1000=3093,53 \\
S X Y=\sum_{i=1}^{n} x_{i} y_{i}=0+292,22 \cdot 240+455,82 \cdot 480+598,09 \cdot 720+ \\
+747,42 \cdot 960+1000 \cdot 1200=2637074,4
\end{gathered}
$$

There is the execution condition (2) to find parameters of $a$ and $b$ :

$$
\left\{\begin{array}{c}
3168000 a+3600 b=2637074,4 \\
3600 a+6 b=3093,55
\end{array}\right.
$$

When solving the system of equations, we receive the coefficient value.

$$
\begin{gathered}
a=\frac{2637074,4 \cdot 6-3093,53 \cdot 3600}{3168000 \cdot 6-3600 \cdot 3600}=0,7747 \\
b=\frac{2209472,71 \cdot 3600-3600 \cdot 2637074,4}{3168000 \cdot 6-3600 \cdot 3600}=50,7438 .
\end{gathered}
$$

Received coefficient values: We insert $a=0.7747$ and $b=50.7438$ into the linear function and check for adequacy of the linear function selected.

Let us calculate values of the approximative function $f=0.7747 x+50.7438$ and enter the received data in the Table 2.

Table 2. Calculated results.

\begin{tabular}{|c|c|c|c|c|c|c|}
\hline $\boldsymbol{n}$ & $\mathbf{1}$ & $\mathbf{2}$ & $\mathbf{3}$ & $\mathbf{4}$ & $\mathbf{5}$ & $\mathbf{6}$ \\
\hline$X$ & 0 & 240 & 480 & 720 & 960 & 1200 \\
\hline$Y$ & 0 & 292.22 & 455.82 & 598.09 & 747.42 & 1000 \\
\hline$F=a x+b$ & 50.74 & 236.68 & 422.62 & 608.56 & 794.50 & 980.44 \\
\hline$\varepsilon_{\mathrm{i}}$ & -50.74 & 55.54 & 33.20 & -10.47 & -47.08 & 19.56 \\
\hline$\delta_{\mathrm{i}}$ & - & 0.1901 & 0.0728 & -0.0175 & -0.0630 & 0.0196 \\
\hline
\end{tabular}

The table shows that deviation of the values of the received theoretical and experimental data is like $Y$ for all points of $X$.It follows that the dependence under study may represented by the linear function $f=0.7747 x+50.7438$.

In order to model the linear approximation of the function in Excel, we add a trendline of linear approximation of the function constructed on the basis of the table of experimental data of the parameters under study. Then we develop the equation of the function and the $\mathrm{R}^{2}$ approximation certainty factor in the diagram's field (fig. 5). 


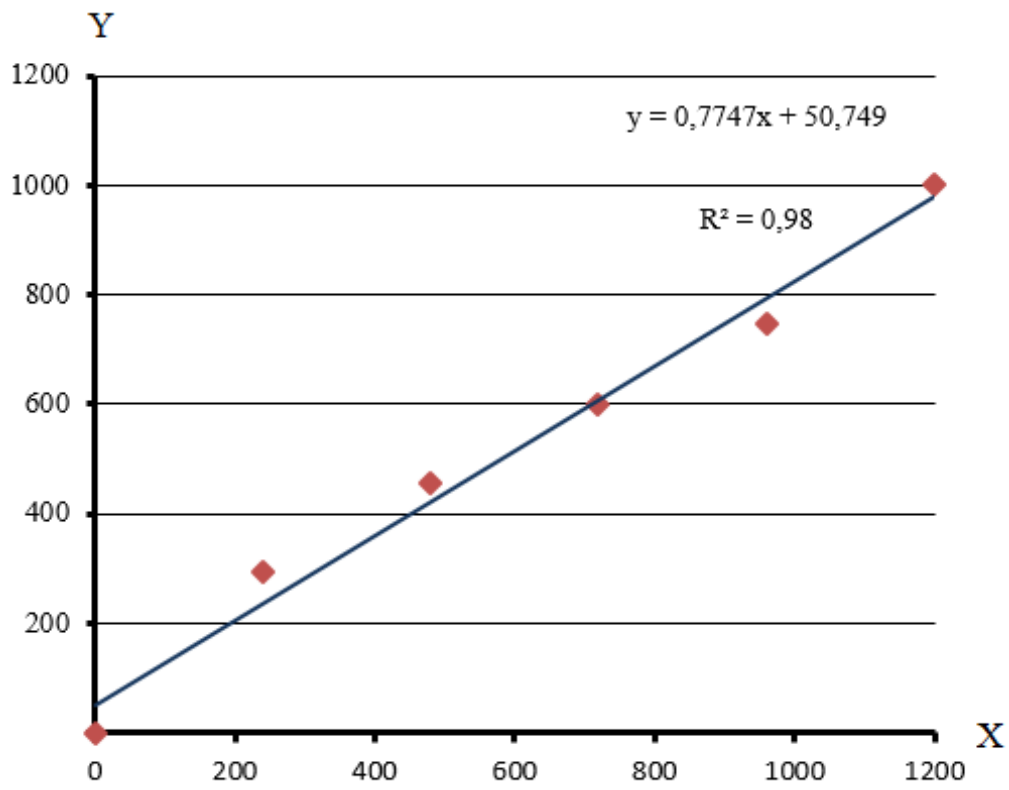

Fig. 5. Linear approximation

In the result of machine modeling of the linear regression, we received coefficient values:We insert $a=0.7747$ and $b=50.749$ into the linear function and receive the approximative function $f=0.7747 x+50.749$

Conclusions.It has been established that the degree of hammers' impact on the threshed mass in a threshing separator designed as hyperboloid of revolution of one nappe rises depending on load.Graphical dependences between humidity of the threshed mass and values of separation and threshing have been determined.

Results of experimental studies have shown that total separation decreases across all zones with the increase in humidity, therefore the threshing process is resistant to humidity changes.

The optimized cutting profile of a transporter, oriented on uniform supply of grain mass, may be represented by a linear model as theoretical data have proved significant validity.

\section{References}

1. I. Antypas, A. Dyachenko, T. Savostina, Journal of Physics: Conference Series 1515(4), 1-10 (2020) doi: 10.1088/1742-6596/1515/4/042051.

2. I.R. Antypas., A.G. Dyachenko, International Journal of Environmental and Science Education 11(18), 10941-10950 (2016)

3. I.R. Antypas., A.G. Dyachenko, T.P. Savostina, IOP Conference Series: Earth and Environmental Science 401(3) (2019) doi:10.1088/1755-1315/403/1/012162.

4. I.R. Antypas, G Kharmanda, A. Dyachenko, T. Savostina, E3S Web of Conferences 116, 00002 (2019) doi: 10.1051/e3sconf/201911600002.

5. A.N. Sirotenko, S.A. Partko, International Journal of Applied Engineering Research 12(14), 4599-4603 (2017) 
6. Y. Tassa, T. Wu, J. Movellan, E.Todorov, IEEE International Conference on Mechatronics and Automation, IEEE ICMA 2013 6617958, 437-443 (2013) doi: 10.1109/ICMA.2013.6617958.

7. A. Sirotenko, S. Partko, Journal of Physics: Conference Series 1399(4) (2019) doi: 10.1088/1742-6596/1399/4/044098.

8. A.N. Sirotenko, S.A. Partko, S.A. Voinash, Lecture Notes in Mechanical Engineering 1325-1333 (2020) doi: 10.1007/978-3-030-22063-1_140.

9. S.A. Partko, L.M. Groshev, A.N. Sirotenko, Lecture Notes in Mechanical Engineering 839-844 (2020) doi: 10.1007/978-3-030-22041-9_89.

10. G. Krytikov, M. Strizhak, V. Strizhak, Eastern-European Journal of Enterprise Technologies 1(7-85), 38-44 (2017) doi: 10.15587/1729-4061.2017.92833.

11. A. Karnoub, H. Huang, I. Antypas, E3S Web of Conferences 175, 12005 (2020)

12. D.G. Aggelis, N. M. Barkoula, T.E Matikas, A.S. Paipetis, Composites Science and Technology 72(10), 1127-1133 (2012) doi: 10.1016/j.compscitech.2011.10.011.

13. K Aniskevich, A. Aniskevich, A. Arnautov, J. Jansons, Composite Structures 94(9), 2914-2919 (2012) doi: 10.1016/j.compstruct.2012.04.030.

14. I.R. Antypas, Journal of Physics: Conference Series 1515(4), 042042 (2020)

15. H. Jin, J. Gonzalez-Gutierrez, P. Oblak, B. Zupančič, I. Emri, Polymer Degradation and Stability 97(11), 2262-2272 (2012) doi: 10.1016/j.polymdegradstab.2012.07.039 